International Journal of Medical Sciences

ISSN 1449-1907 www.medsci.org 2006 3(4):141-147

Research Paper

(c)2006 Ivyspring International Publisher. All rights reserved

\title{
A possible link between exercise-training adaptation and dehydroepiandros- terone sulfate- an oldest-old female study
}

\author{
Yi-Jen Huang ${ }^{1}$, Mu-Tsung Chen ${ }^{2}$, Chin-Lung Fang ${ }^{3}$, Wen-Chih Lee ${ }^{4}$, Sun-Chin Yang ${ }^{2}$, Chia-Hua Kuo ${ }^{2}$ \\ 1. Department of Kinesiology, SooChow University, Taipei, Taiwan \\ 2. Laboratory of Exercise Biochemistry, Taipei Physical Education College, Taipei, Taiwan \\ 3. Department of Kinesiology, National Normal Taiwan University, Taipei, Taiwan \\ 4. Committee of General Studies, Shih Hsin University, Taipei, Taiwan
}

Correspondence to: Chia-Hua Kuo, Ph.D., Laboratory of Exercise Biochemistry, Taipei Physical Education College, 5 Dun-Hua N. Rd, Taipei, Taiwan 105. Phone: +886-2-25774624 ext. 831, Fax: +886-2-25790526, E-mail: kch@tpec.edu.tw

Received: 2006.08.04; Accepted: 2006.09.10; Published: 2006.09.10

The purpose of this study was to determine the association between the level of salivary dehydroepiandrosterone sulfate (DHEA-S) and the magnitude of adaptation to exercise training in insulin sensitivity for aged females. A group of 16 females, aged 80-93 years old, was divided into 2 groups according to their baseline DHEA-S levels: Lower Halves $(\mathrm{N}=8)$ and Upper Halves $(\mathrm{N}=8)$, and participated in a 4-month exercise intervention trial. Insulin response with an oral glucose tolerance test (OGTT), cholesterol, blood pressure (BP), motor performance, and DHEA-S were determined at baseline and 4 months after the training program. Glucose tolerance and body mass index (BMI) remained unchanged with training for both groups. Insulin, fasted cholesterol, diastolic blood pressure, reaction time, and locomotive function were significantly lowered by training only in the Upper Halves group. Changes in the area under curve of insulin (IAUC) were negatively correlated with the baseline DHEA-S level $(\mathrm{R}=-0.60, P<0.05)$. The current study provides the first evidence that oldest-old subjects with low DHEA-S level appear to be poor responders to exercise-training adaptations.

Key words: Cholesterol, triglycerides, oldest-old, motor performance, blood pressure

\section{Introduction}

Insulin resistance is considered a common pathogenic origin to several age-associated metabolic disorders [1, 2]. It is presently known that the reduction in insulin sensitivity, characterized by exaggerated glucose and insulin responses under glucose challenge, occur progressively with advancing age [3, 4]. While numerous studies confirm the benefit of regular exercise training on improving insulin sensitivity and glucose tolerance in human [5], it was also reported that the exercise training effect on improving insulin sensitivity and glucose tolerance is not as effective in middle-aged or older groups as in young people [6]. The physiologic mediator essential for the exercise training effect observed in young individuals but vanished in the elderly is currently unknown.

DHEA-S is one of the steroids known to decrease with age [7], and has recently been suggested as a biomarker of human aging [8]. Comparing cumulative survival percentages of healthy men in the Baltimore Longitudinal Study of Aging (BLSA) who are divided into two groups according to the insulin and DHEA-S levels, it was shown that individuals with lower insulin and higher DHEAS have greater survival than respective counterparts [9]. Low concentrations of DHEA-S are associated with an increased risk in cardiovascular disease $[10,11]$ and diabetes $[12,13]$. The causal relationship between DHEA-S and insulin action is recently supported by the evidence that in- creasing serum DHEA-S with exogenous DHEA supplementation significantly enhances insulin sensitivity in elderly [14].

Exercise training can be considered a type of stress that is known to induce a number of metabolic changes. It has been generally thought that survival and longevity is associated with successful adaptation against environmental stress. DHEA-S has been documented to have a buffering action against stress $[15,16]$ and plays a role in functional recovery in humans [17]. To determine the association of DHEA-S with exercise-training adaptation, the effect of 4 months of exercise training on insulin resistance measures was determined in a group of oldest-old females dichotomized into Lower Halves and Upper Halves according to their baseline DHEA-S levels.

\section{Materials and Methods}

Human Subjects

Institutionalized female subjects (Taipei, Taiwan), aged 80 years and older, with living dependence were recruited for a health promotion program by posters. Exclusion criteria were musculo-skeletal disorders and cognitive or physical dysfunction interfering with test and training procedures. All subjects demonstrated a capability of finishing a continuous 6 minute walk. 16 females were eligible and were equally divided into two groups according to their baseline (pre-trained) saliva DHEA-S level: Lower Halves $(\mathrm{N}=8$, age 
$83.5 \pm 0.60$ years, DHEA-S $<2.2 \mathrm{ng} / \mathrm{mL}$ ) and Upper Halves groups $(\mathrm{N}=8$, age $83.9 \pm 0.85$ years, DHEA-S > $2.2 \mathrm{ng} / \mathrm{mL}$ ). The median value of pre-trained DHEA-S levels in the 16 women is $2.2 \mathrm{ng} / \mathrm{mL}$. The normal reference ranges for salivary DHEA-S are 0.69-15.7 $\mathrm{ng} / \mathrm{mL}$ (healthy young female, $\mathrm{N}=12$ ) and $0.98-16.7$ $\mathrm{ng} / \mathrm{mL}$ (healthy young male, $\mathrm{N}=9$ ). Therefore, DHEA-S level for the Lower Halves groups can be considered extremely low. Aims and methods were explained to all subjects, who then signed a formal consent. This work was conducted in accordance with the guidelines in the Declaration of Helsinki. Ethical approval for the study was obtained from the Human Subject Committee of Taipei Physical Education College.

\section{Exercise training program}

The exercise program complied with the ACSM's Active Aging Partnership and the Strategic Health Initiative on Aging guidelines, which includes walking and resistive exercise using their own body weight. All subjects walked briskly for 20 minutes at $45-80 \%$ heart rate (HR) reserve (to achieve an intensity of 11-14 of Rating of Perceived Exertion Scale), more than 5 days a week. They also performed a 30 minute resistance training (3 times per week) consisting of a 5-minute warm-up and a 5-minute cool-down period of low-intensity dynamic exercise involving concentric and eccentric contractions. Exercise used for the training included squat, leg extension, upright row, lateral pull-down, standing leg curls (ankle weights), and abdominal curls. All subjects were required to perform each repetition in a slow, controlled manner, with a rest of 2-3 minutes between sets. One or two sets of 12-15 repetitions were performed for all exercises at each training session. All sessions were supervised to ensure safety and correct techniques and to monitor the appropriate amount of exercises and rest intervals. Saliva and blood samples were taken before and after the 4-month exercise training program (18 hours after the last bout of exercise), in the morning under fasted condition (8-9 am).

\section{Saliva DHEA-S level}

Approximately $1 \mathrm{ml}$ of saliva was collected in a container, using a plastic straw. A $100 \mu \mathrm{l}$ aliquot of saliva samples and standards $(0,0.1,0.3,1,5,10,30$ $\mathrm{ng} / \mathrm{mL}$ ) were used for DHEA-S determination. DHEA-S was quantified by ELISA using a commercial DHEA-S (Saliva) EIA ELISA kit (DSL-10-2700S, Diagnostic System Laboratories, Webster, Texas, USA). The assay procedure was performed according to the manufacture's instructions. Performance characteristics of the saliva DHEA-S assay, including sensitivity, specificity, precision, and recovery, and linearity, are reported on the manufacture's instructions.

\section{Oral Glucose Tolerance Test (OGTT) and insulin re-} sponse

A 75-gram bolus of glucose was orally delivered with $500 \mathrm{ml}$ of pure water. Blood samples were collected from the fingertips at 0 (fasted value), 30, 50, and 80 minutes. A glucose analyzer (Lifescan, California, USA) was utilized for glucose determination. A serum sample was collected from $200 \mu$ of fingertip blood and used for insulin determination [18]. Serum insulin levels were determined on an ELISA analyzer (Tecan Genios, Salzburg, Austria) with the use of commercially available ELISA kits (Diagnostic Systems Laboratories, Inc. Webster, Texas, USA), according to the manufacture's instructions.

\section{Serum triglyceride and cholesterol levels}

Total cholesterol and triglyceride were measured on a Beckman spectrophotometer analyzer following the Sigma Trinder's reaction (Sigma, Missouri, USA), according to the manufacturer's procedures.

\section{$B P, H R$, and arterial oxygen saturation}

$\mathrm{BP}$ and HR were measured quietly and at constant temperature $\left(\sim 23^{\circ} \mathrm{C}\right)$. Participants were provided with an automated oscillometric BP monitor (Oscar-1; SunTech Medical Instruments, Inc., Raleigh, NC, USA) with the arm cuff secured on the upper left arm while arterial oxygen saturation arterial oxygen saturation $\left(\mathrm{SaO}_{2}\right)$ and $\mathrm{HR}$ were measured on the right hand using a $\mathrm{MAXO}_{2}$ monitor (Maxtec Inc, Salt Lake City, Utah, USA).

\section{Motor performances}

Locomotive function was assessed by measuring the time it took to complete walking around two cones and the distance walked in 6 minutes. Visuomotor response time was measured by recording hand-reaction time and foot tapping as motor processing [19].

\section{Statistical Analysis}

Two-way analysis of variance with repeated measures was used to compare the mean differences between all measured values before and after the exercise training for both groups. Fisher's protected least significance test, which holds the value of a type I error constant for each test, was utilized to distinguish the significant differences between pairs of groups. Regression analysis was performed for the changes in AUC (glucose and insulin) with exercise training and baseline DHEA-S level. The power for the regression analysis was 0.88 with 16 subjects. A level of $P<0.05$ was set for significance for all tests. All values are expressed as means \pm standard errors. SPSS 10.0 was used for the statistical analysis.

\section{Results}

Physical characteristics of the subjects and their baseline saliva DHEA-S level (pre-trained value) are shown in Table 1. The 4-month exercise training did not affect body weight, BMI, and DHEA-S in both female groups. The DHEA-S level in the Lower Halves was extremely low compared to healthy young females (reference range values are shown in Methods). The Upper Halves subjects displayed a significantly shorter statue compared to those Lower Halves subjects $(P<0.05)$. The BMI of the Upper Halves group 
was significantly greater than that of the Lower Halves group.

Glucose tolerance and insulin response are shown in Figure 1 (for Lower Halves group) and Figure 2 (for Upper Halves group). The current exercise training program did not significantly alter fasted and postprandial glucose levels for both the Lower Halves and Upper Halves groups. Fasted insulin levels in both groups were not significantly affected by exercise training. Under glucose challenge conditions, insulin levels in the Upper Halves group were significantly lowered by the exercise-training program (50 th and $\left.80^{\text {th }} \mathrm{min}, P<0.05\right)$, whereas no change was found in the Lower Halves group. Similarly, serum cholesterol levels in the Upper Halves group were significantly lowered by exercise training $(P<0.05)$, but not in the Lower Halves group. The exercise training significantly lowered fasted triglyceride in both groups $(P<$ $0.05)$. Fasted glucose and insulin levels between the Upper Halves and Lower Halves groups were not different $(P<0.05)$. Fasted triglyceride and cholesterol levels between the Upper Halves and Lower Halves groups were not different. Figure 3 shows the relationships between baseline (pre-trained) DHEA-S level and changes in the area under curve of glucose (GAUC, Figure 3A) and insulin (IAUC, Figure 3B) by training. GAUC change did not significantly correlate with DHEA-S, whereas the IAUC change was negatively correlated with DHEA-S $(\mathrm{R}=-0.60, P<0.05)$.

Effects of the 4-month exercise training on cardiovascular variables are displayed in Table 2. Exercise training did not affect systolic BP for both groups. Diastolic BP and resting HR were significantly lowered by exercise training only in the Upper Halves group $(P<0.05)$. Resting $\mathrm{SAO}_{2}$ for both groups was not affected by exercise training. These cardiovascular variables between the Upper Halves and Lower Halves groups were not significantly different.

Data for motor performance measures are shown in Table 3. Exercise training significantly improved visuomotor response time and locomotion/agility only in the Upper Halves group $(P<0.05)$. The training program did not significantly affect the 6 minute walking performance for eitehr group.

\section{Discussion}

It was reported that the exercise-training effect on improving insulin sensitivity and glucose tolerance in middle-aged or older individuals is not as effective as it is in young individuals [6]. The physiologic mediator conveying the exercise training effect that diminished with age is currently unknown. In this study we examined the effect of a 4-month exercise program on glucose tolerance and insulin sensitivity in a group of females aged 80-93 years, in relation to their baseline DHEA-S levels. We found that the normal exercise-training effect on improving insulin sensitivity was absent in the Lower Halves of DHEA-S subjects. The basal DHEA-S level of this group $(0.60 \mathrm{ng} / \mathrm{mL})$ is considered extremely low compared to healthy young females $(0.69-15.7 \mathrm{ng} / \mathrm{mL})$. The current study demonstrates that the oldest-old subjects with low DHEA-S level were poor responders to exercise-training adaptation.

Age is a well-recognized risk factor for insulin resistance syndromes [4], which includes a clustering of interrelated plasma lipid and BP abnormalities [1, 2, 20]. The causal relationship between $B P$ and insulin sensitivity has already been demonstrated elsewhere $[2,22]$. Here we found that the reductions in diastolic $\mathrm{BP}$ and cholesterol by exercise training were apparent in the Upper Halves of DHEA-S, but not for the Lower Halves of DHEA-S. Combination of high BP and cholesterol level is known as a major risk factor leading to stroke. The involvement of DHEA-S in the exercise-training effect on cholesterol level is also supported by Yang's study [18], in which exercise training combined with exogenous DHEA supplementation resulted in a 3-fold increase in serum DHEA-S and enhanced the cholesterol-lowering effect of exercise training. Previous studies regarding the exercise training on this cholesterol-lowering effect remain inconsistent [21]. According to the present results, individual variations in DHEA-S level can be one possibility that accounts for the discrepancy among studies.

Another important finding of this study is that the oldest-old females with greater DHEA-S levels exhibited greater enhancement in motor performance. This result could be related to the improvements in both muscular and neuronal components secondary to the improvement in insulin sensitivity. Increasing peripheral insulin action could result in better capability to store glycogen [23] and a reduced rate of muscle protein degradation [24]. This effect is beneficial in preserving greater anaerobic fuel and normal contractile property of skeletal muscle in response to acute physical challenge. In addition, aged individuals are usually faced with the problem of poor insulin sensitivity and an increased risk of developing type 2 diabetes, which can have major impacts on nutrient supplies for peripheral motor neurons due to microvascular defects [27]. DHEA-S is also known as a neuroactive steroid [25] that has been found to exert a neuroprotective effect on motor neurons, as evidenced by the fact that supplementing the diet with DHEA for more than 5 week prevents the diabetes-induced development of neural dysfunction [26].

Improvement in carbohydrate metabolism by exercise training may be functionally relevant to survival and longevity. It has previously been shown that environmental stress is persistently occurring throughout the entire lifetime and threatens human survival. Insufficient adaptation against stress in older age with concomitant reductions in DHEA-S may be linked to the decrease in cumulative survival of humans [9]. Under stress conditions, ATP demands are immediately increased. Carbohydrate fuel, as an anaerobic substrate, has the advantage of having a fast degradation rate and can occur in the absence of oxygen for rapid ATP resynthesis. Therefore, carbohydrate storage becomes crucial for survival under acute stress when the increasing oxygen delivery system takes longer to be fully recruited for fatty acid oxida- 
tion. Exercise is a known stress condition that consumes muscle glycogen rapidly. During the recovery period following exercise, the whole-body glucose tolerance and the rate of muscle glycogen storage increases simultaneously, resulting in glycogen supercompensation [5]. This normal adaptation scheme ensures that the human body reserves more carbohydrate fuel for better coping capability in the recurrence of a similar challenge.

A number of recent studies suggest that DHEA-S may be essential for physiologic adaptation against environmental stress $[9,18,28,29]$ and thus relevant to survival and longevity in humans. Roth et al [9] has found that age-dependent DHEA-S declines were paralleled with reduced cumulative survival in the human population and those individuals with an earlier decline in DHEA-S exhibited lower average life expectancy. A recent study by Tsai et al [28] demonstrated that an acute bout of exercise challenge resulted in a pronounce decline in DHEA-S levels of young male athletes during the recovery period. Lee et al [29] has also found a similar trend of DHEA-S decline in the young subjects with higher DHEA-S level during a prolonged mountaineering activity, whereas the subjects with lower DHEA-S appeared to have no room for decline and displayed poor adaptation. In particular, the normal physiologic adaptation to the high altitude activity, including increase in red blood cell concentration and improvement in insulin sensitivity for glucose uptake, was absent in the subject with lower baseline DHEA-S levels. In addition, these low DHEA-S young subjects appear to be poor-responders to endogenous erythropoietin (EPO), as they exhibited greater EPO level at sea-level and altitude but exhibited an insignificant increase in red blood cell by prolonged altitude activity. Therefore, DHEA-S decline is likely due to an increased demand for physiologic adaptation.

In this study, the BMI values are different between the Upper and Lower Halves of the DHEA-S. It is thus possible that the observed exercise-training effects are simply due to the influenced of weight status. However, while insulin sensitivity and motor performance were improved in the Upper Halves, the BMI for both groups was not altered by the 4-month exercise training program. Additionally, in Lee's study, physiologic acclimatization of young healthy subjects was also lower in the subject with lower DHEA-S level [29], while BMI for the high and low DHEA-S groups was not different. Therefore, initial weight status is unlikely to contribute to the different training response of the two groups in this study. Conversely, low BMI in this age group may be caused by low DHEA-S production [30].

In conclusion, this study demonstrates that the oldest-old subjects with low DHEA-S level are poor responders to exercise-training adaptation. The effects of exercise training on improving insulin resistance measures, including insulin sensitivity (IAUC), cholesterol, and BP, were absent in the subjects with low DHEA-S levels. Additionally, the oldest-old females with lower DHEA-S gained fewer benefits on enhancing motor performance from exercise training.

\section{Acknowledgements}

This research was supported by the National Science Council, ROC, Grant NSC93-2413-H031-004.

\section{Conflict of interests}

The authors have declared that no conflict of interest exists.

\section{References}

1. Facchini FS, Hua N, Abbasi F, et al. Insulin resistance as a predictor of age-related diseases. J. Clin. Endocrinol. Metab. 2001; 86: 3574-8.

2. Reaven GM. Role of insulin resistance in human disease. Diabetes. 1988; 37: 1595-1607.

3. Davidson MB. The effect of aging on carbohydrate metabolism: a review of the English literature and a practical approach to the diagnosis of diabetes mellitus in the elderly. Metabolism. 1979; 28: 688-705.

4. Paolisso G, Rizzo MR, Mazziotti G, et al. Advancing age and insulin resistance: role of plasma tumor necrosis factor-alpha. Am J Physiol. 1998; 275: E294-9.

5. Ivy JL, Zderic TW, Fogt DL. Prevention and treatment of non-insulin-dependent diabetes mellitus. Exerc Sport Sci Rev. 1999; 27: 1-35.

6. Short KR, Vittone JL, Bigelow ML, et al. Impact of aerobic exercise training on age-related changes in insulin sensitivity and muscle oxidative capacity. Diabetes. 2003; 52: 1888-96.

7. Orentreich N, Brind JL, Rizer RL, et al. Age changes and sex differences in serum deydroepiandrosterone sulfate concentrations throughout adulthood. J Clin Endocrinol Metab. 1984; 59: 551-5.

8. Lane MA, Ingram DK, Ball SS, et al. Dehydroepiandrosterone sulfate: a biomarker of primate aging slowed by calorie restriction. J Clin Endocrinol Metab. 1997; 82: 2093-6.

9. Roth GS, Lane MA, Ingram DK, et al. Biomarkers of caloric restriction may predict longevity in humans. Science. 2002; 297: 811.

10. Kroboth PD, Salek FS, Pittenger AL, et al. DHEA and DHEA-S: a review. J Clin Pharmacol. 1999; 39: 327-48.

11. Nafziger AN, Herrington DM, Bush TL. Dehydroepiandrosterone and dehydroepiandrosterone sulfate: Their relation to cardiovascular disease. Epidemiol Rev. 1991; 13: 267-93.

12. Slowinska-Srzednicka J, Malczewska B, Srzednicki M, et al. Hyperinsulinemia and decreased plasma levels of dehydroepiandrosterone sulfate in premenopausal women with coronary artery disease. J Intern Med. 1995; 237: 465-72.

13. Vermeulen A. Decreased androgen levels and obesity in men. Ann Med. 1996; 28: 13-5.

14. Villareal DT, Holloszy JO. Effect of DHEA on abdominal fat and insulin action in elderly women and men: a randomized controlled trial. JAMA. 2004; 292: 2243-8.

15. Cruess DG, Antoni MH, Kumar M, et al. Cognitive-behavioral stress management buffers decreases in dehydroepiandrosterone sulfate (DHEA-S) and increases in the cortisol/DHEA-S ratio and reduces mood disturbance and perceived stress among HIV-seropositive men. Psychoneuroendocrinology. 1999; 24: 537-49.

16. Regelson W, Loria R, Kalimi M. Hormonal intervention: "buffer hormones" or "state dependency". The role of dehydroepiandrosterone (DHEA), thyroid hormone, estrogen and hypophysectomy in aging. Ann N Y Acad Sci. 1988; 521: 260-73.

17. Herbert J. Neurosteroids, brain damage, and mental illness. Exp Gerontol. 1998; 33: 713-27. 
18. Yang SC, Chen CY, Liao YH, et al. Interactive effect of an acute bout of resistance training and DHEA administration on glucose tolerance and serum lipids in middle-aged women. Chin J Physiol. 2005; 48: 23-29.

19. Shigematsu R, Chang M, Yabushita N, et al. Dance-based aerobic exercise may improve indices of falling risk in older women. Age Ageing. 2002; 31: 261-266.

20. Krauss RM. Lipids and lipoproteins in patients with type 2 diabetes. Diabetes Care 2004; 27: 1496-504.

21. Marti B. Health effects of recreational running in women. Some epidemiological and preventive aspects. Sports Med. 1991; 11: 20-51.

22. Martinez FJ, Rizza RA, Romero JC. High-fructose feeding elicits insulin resistance, hyperinsulinism, and hypertension in normal mongrel dogs. Hypertension 1994; 23: 456-63.

23. Jensen J, Ruzzin J, Jebens E, et al. Improved insulin-stimulated glucose uptake and glycogen synthase activation in rat skeletal muscles after adrenaline infusion: role of glycogen content and PKB phosphorylation. Acta Physiol Scand. 2005; 184: 121-30.

24. Tischler ME, Satarug S, Aannestad A, et al. Insulin attenuates atrophy of unweighted soleus muscle by amplified inhibition of protein degradation. Metabolism 1997; 46: 673-9.

\section{Tables and Figures}

Table 1. Physical characteristics of the oldest-old subjects before (Pre) and after 4-month exercise training (Post). \# significance against Lower Halves group of DHEA-S, $P<0.05$. Data are divided into upper and lower halves according to baseline DHEA-S values for better comparison with the two groups.

\begin{tabular}{ccccc} 
& \multicolumn{2}{c}{ Lower Halves } & \multicolumn{2}{c}{ Upper Halves } \\
& Pre & Post & Pre & Post \\
Age (year) & $83.5 \pm 0.60$ & - & $83.9 \pm 0.85$ & - \\
Height (centimeter) & $154.8 \pm 1.6$ & $153.9 \pm 1.4$ & $149.8 \pm 1.9 \#$ & $149.0 \pm 1.7 \#$ \\
Weight $(\mathrm{kg})$ & $56.7 \pm 2.7$ & $56.7 \pm 2.8$ & $61.9 \pm 4.5$ & $60.4 \pm 5.0$ \\
BMI & $23.8 \pm 1.4$ & $24.0 \pm 1.4$ & $27.5 \pm 1.6 \#$ & $27.0 \pm 1.7 \#$ \\
DHEA-S (ng/mL) & $0.6 \pm 0.3$ & $2.3 \pm 0.3$ & $7.5 \pm 2.7 \#$ & $5.0 \pm 2.3 \#$ \\
\hline
\end{tabular}

Table 2. Cardiovascular risk factors of the oldest-old subjects before (Pre) and after 4-month exercise training (Post). * significance against Pre, $P<0.05$. \# significance against Lower Halves group of DHEA-S, $P<0.05$. Data indicates that diastolic BP and total cholesterol were lowered by exercise training only in the oldest-old subjects with greater DHEA-S level.

\begin{tabular}{ccccc} 
& \multicolumn{2}{c}{ Lower Halves } & \multicolumn{2}{c}{ Upper Halves } \\
& Pre & Post & Pre & Post \\
Systolic BP (mmHg) & $130 \pm 4.7$ & $127 \pm 5.4$ & $133 \pm 2.6$ & $126 \pm 2.7$ \\
Diastolic BP (mmHg) & $71 \pm 1.5$ & $67 \pm 2.3$ & $77 \pm 3.5 \#$ & $68 \pm 1.9^{*}$ \\
Resting HR & $72 \pm 3.3$ & $70 \pm 3.4$ & $76 \pm 3.5$ & $69 \pm 2.5^{*}$ \\
Arterial oxygen saturation (\%) & $98 \pm 0.5$ & $97 \pm 0.4$ & $99 \pm 0.2$ & $97 \pm 0.4$ \\
Fasted triglycerides (mg/dl) & $152 \pm 38.8$ & $84 \pm 16.7^{*}$ & $121 \pm 13.5$ & $85 \pm 7.8^{*}$ \\
Fasted cholesterol (mg/dl) & $206 \pm 6.5$ & $200 \pm 9.8$ & $196 \pm 6.1$ & $183 \pm 5.1^{*}$
\end{tabular}

Table 3. Motor performance of the oldest-old subjects before (Pre) and after 4-month exercise training (Post). * significance against Pre, $P<0.05$. Visuomotor response and agility were improved by exercise training only in the oldest-old subjects with greater DHEA-S level. Visuomotor response time was measured by recording hand-reaction time and foot tapping as motor processing. Locomotive function assessment was measured by walking time around two cones from seat and 6-minute walking distance.

\begin{tabular}{ccccc}
\hline & \multicolumn{2}{c}{ Lower Halves } & \multicolumn{2}{c}{ Upper Halves } \\
& Pre & Post & Pre & Post \\
Distance for 6-min walk (meter) & $384 \pm 28$ & $351 \pm 44$ & $349 \pm 19$ & $382 \pm 36$ \\
Reaction time (second) & $0.91 \pm 0.06$ & $0.74 \pm 0.13$ & $1.01 \pm 0.11$ & $0.66 \pm 0.07^{*}$ \\
Locomotion time (second) & $10.5 \pm 1.9$ & $8.1 \pm 1.9$ & $10.6 \pm 1.4$ & $8.4 \pm 0.8^{*}$ \\
\hline
\end{tabular}


Figure 1. Glucose tolerance and insulin response in Lower Halves group of DHEA-S before (Pre) and after 4-month exercise training (Post). Glucose levels (A) and insulin level (B) were measured under 75 grams of oral glucose challenge. Glucose tolerance and insulin response were not changed by exercise training in the oldest-old subjects with lower DHEA-S level, suggesting insulin sensitivity was not improved by exercise training.

A

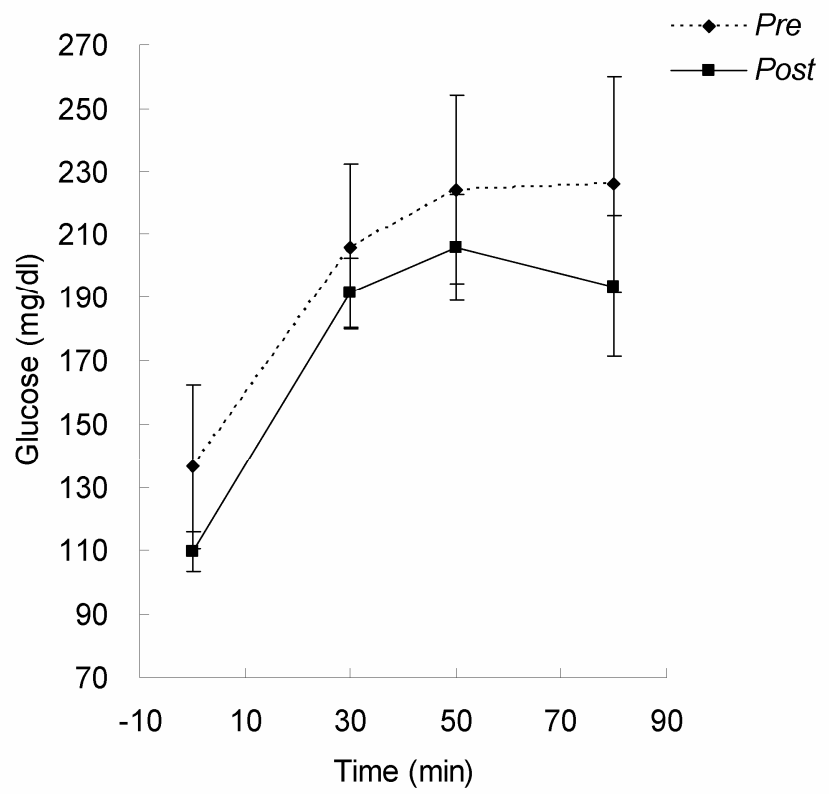

B

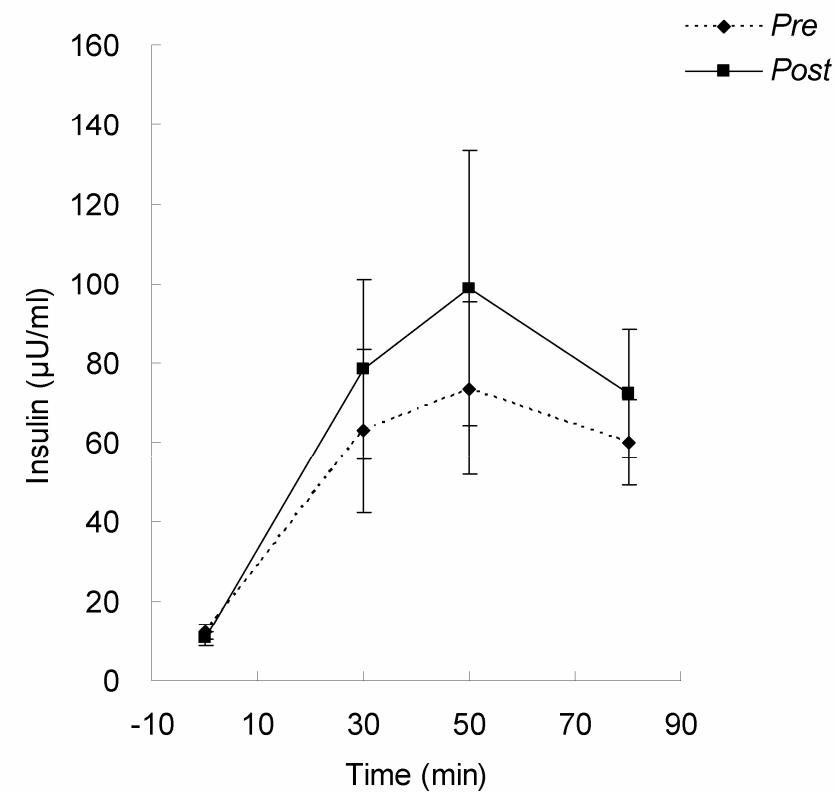

Figure 2. Glucose tolerance and insulin response in Upper Halves group before (Pre) and after 4-month exercise training (Post). Glucose (A) and insulin (B) levels were measured following a 75 gram bolus of oral glucose challenge. * significant difference from Pre $(P<0.05)$. While glucose tolerance remained unchanged, insulin response was lowered by exercise training in the subjects with higher DHEA-S level, suggesting that insulin sensitivity was improved in the oldest-old subjects with higher DHEA-S.

A

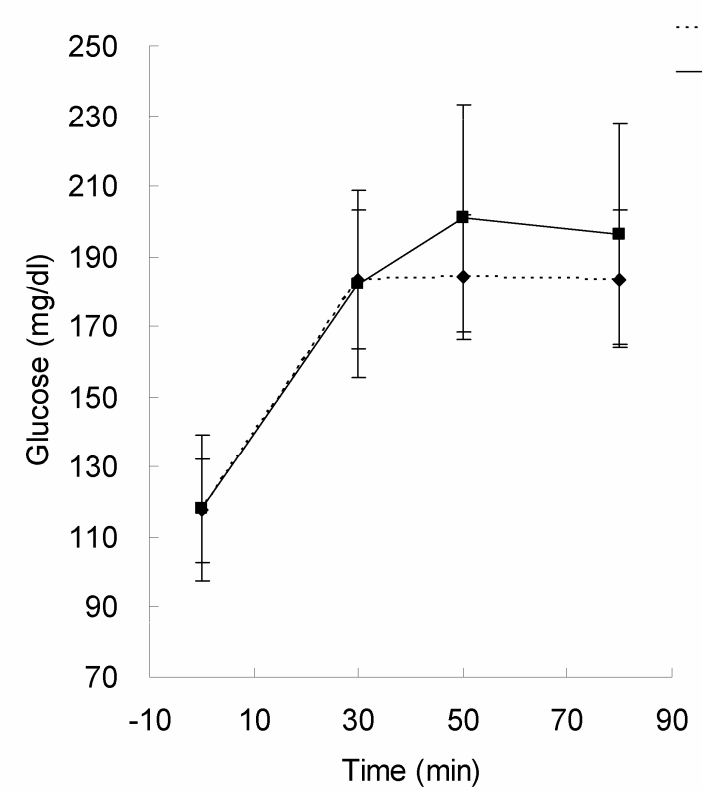

B

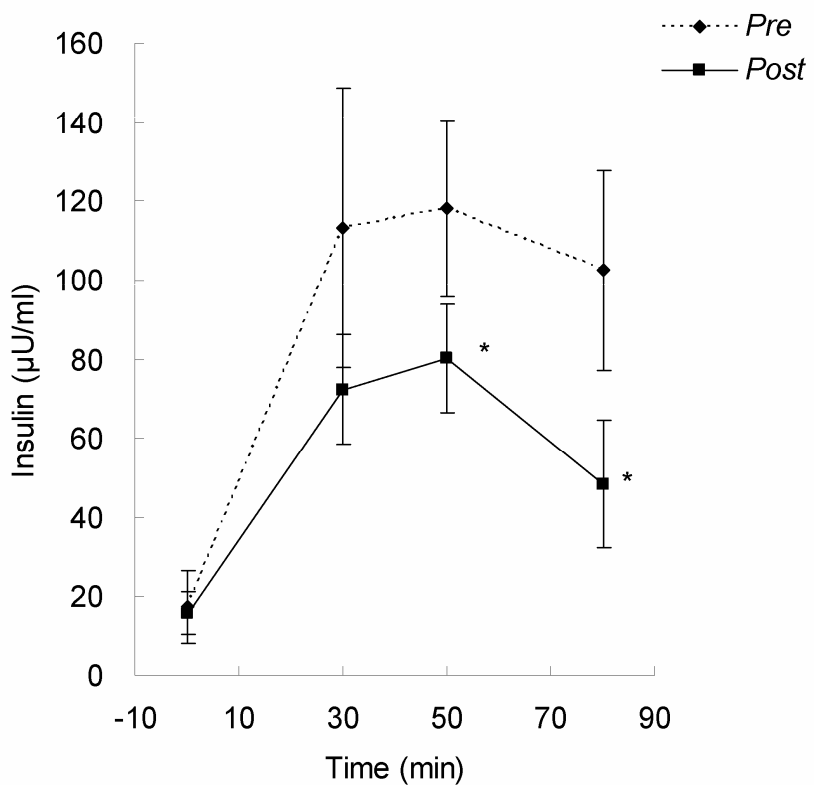


Figure 3. Relationship between baseline DHEA-S level and changes in the area under curve for glucose (GAUC change) and insulin (IAUC change) in the oldest-old subjects. No correlation between GAUC change and baseline DHEA-S level was found (3A); IAUC change was negatively correlated with baseline DHEA-S level (3B) $(\mathrm{R}=-0.60, P<0.05)$. This result indicates that the magnitude of exercise training effect on insulin sensitivity is associated with DHEA-S level.

A.

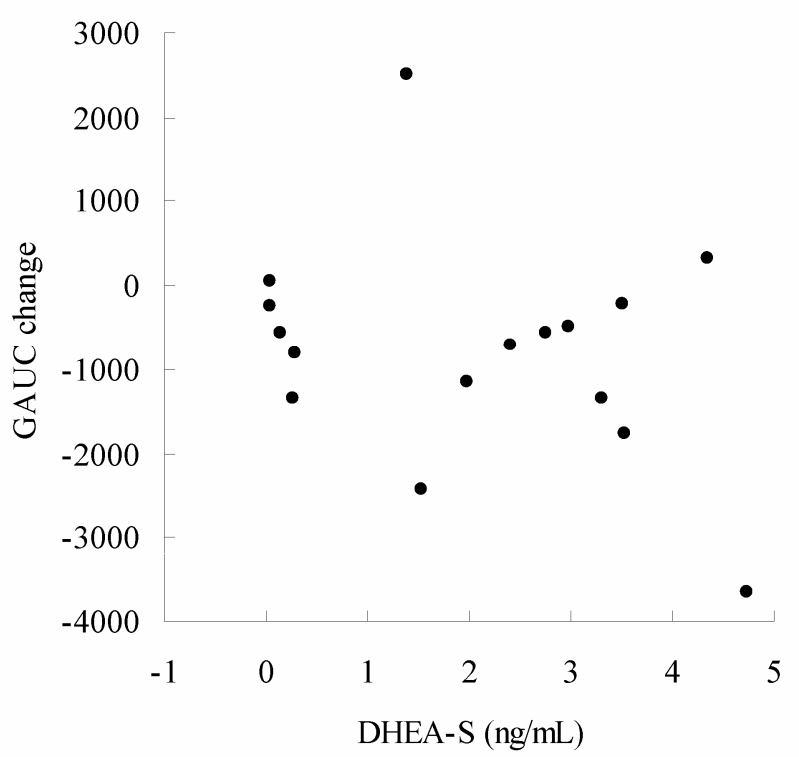

B.

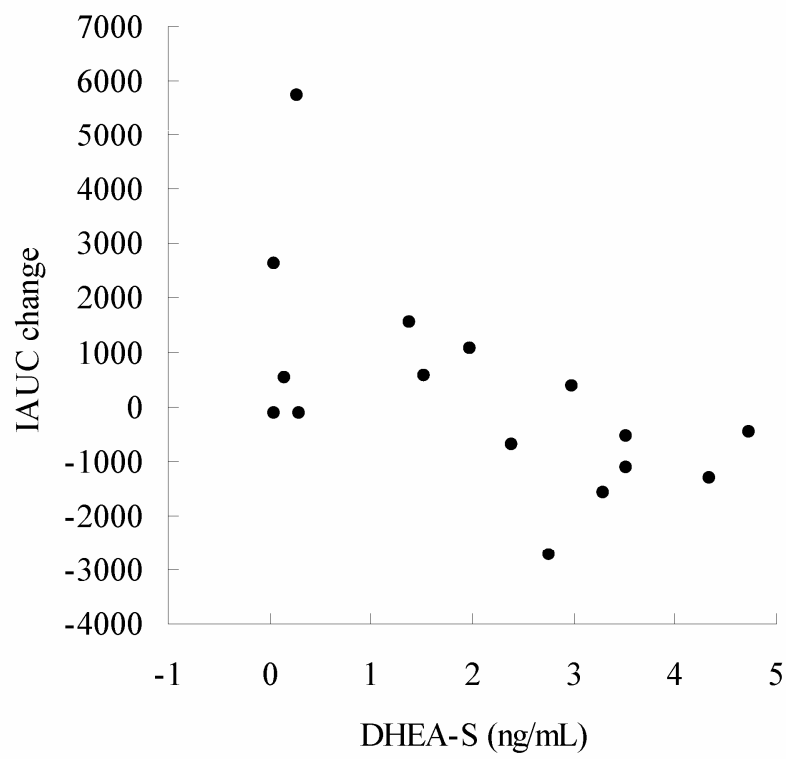

